\title{
Premature Rupture of Membranes Characteristics, Determinants, and Outcomes of in Benghazi, Libya
}

\author{
Milad M. M. Gahwagi*, Musa 0. Busarira, Mona Atia \\ Department of Obstetrics \& Gynaecology, Benghazi Medical University, Benghazi, Libya \\ Email: "
}

Received 19 July 2015; accepted 22 August 2015; published 26 August 2015

Copyright (C) 2015 by authors and Scientific Research Publishing Inc.

This work is licensed under the Creative Commons Attribution International License (CC BY). http://creativecommons.org/licenses/by/4.0/

(c) (i) Open Access

\section{Abstract}

Premature rupture of membranes (PROM) is a frequent phenomenon that occurs in about $4 \%$ 7\%. However, the associated sequent problems are proportionally high. Almost two thirds of PROM are spontaneous, common risk factor young mother, multiple pregnancy, genital tract infection, previous preterm delivery. The complications range from induction of labour, caesarean section, fetal pulmonary morbidity and even mortality. Objectives of the Study: To identify and quantify the risk factors and complications of premature rupture of membrane in pregnant women in Benghazi, Libya, 2012. Material and Methods: 100 pregnant women diagnosed as PROM were examined for the physical signs. Detailed gynaecological and obstetric history was reported. Laboratory investigations included cervical swabs and posterior fornix swabs under aseptic conditions Drugs including types of antibiotics, Dexamethasone and tocolytics were also included. Results: The mean age of 100 patients was 28.9 years. Most of them, $66.1 \%$ of patients received antibiotis mostly Erythromycin. Dexamethasone was given for $32.1 \%$. FTND accounted for $62 \%$ and PTND for $10 \%$ and Caesarean section was performed for $28 \%$. The main indications for C/S were failed induction, in $50 \%, 11.8 \%$ were equally distributed between; macrosomia, previous scars, drained liquor, previous C/S, breech presentation. Only $1 \%$ of new born babies had complication. Conclusions and Recommendations: Further large studies should be done for more elaboration and analytical studies to quantify the strength of each risk factor. Strategy for management of PROM should be adopted for proper timing of induction, emergency caesarean intervention with ultimate goals of minimizing and or preventing maternal and fetal complications of disease.

\section{Keywords}

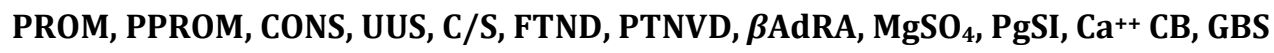

\footnotetext{
${ }^{*}$ Corresponding author.
}

How to cite this paper: Gahwagi, M.M.M., Busarira, M.O. and Atia, M. (2015) Premature Rupture of Membranes Characteristics, Determinants, and Outcomes of in Benghazi, Libya. Open Journal of Obstetrics and Gynecology, 5, 494-504. 


\section{Introduction}

\subsection{Prom}

Premature rupture of membranes (PROM) is defined as a spontaneous leakage of amniotic fluid from the amniotic sac where the baby swims. The fluid escapes through ruptured fetal membranes, occurring after 28 weeks of gestation and at least one hour before the onset of true labour. PROM can occur before or after 40 weeks gestation period. So, the word "premature" does not mean that the gestational age of the fetus is preterm [1]. The final diagnosis of spontaneous rupture of the membranes is best achieved by maternal history followed by a sterile speculum examination. Ultrasound examination is useful in some cases to help confirm the diagnosis. Digital vaginal examination should be restricted if preterm pre-labour rupture of membranes (PPROM) is suspected [2].

\section{A) Classifications of PROM [3]}

Premature PROM occurs after twenty-eight weeks of gestational age and before thirty-seven weeks. Term RPOM occurs after thirty-seven completed weeks of gestational age, including post-term cases occurring after forty weeks. Preterm PROM and term PROM are further divided into:

Early PROM (less than twelve hours has passed since the rupture of fetal membranes).

Prolonged PROM (twelve or more hours has passed since the rupture of fetal membranes).

\section{B) Risk Factors for PROM}

Rupture of the fetal membranes can occur when the cervix is either closed or dilated. Sometimes, it can occur in a very early pregnancy (before twenty-eight weeks-this leads to inevitable abortion), or in early third trimester (between twenty-eight and thirty-four weeks). Risk factors highly associated with PROM: Infection, Malpresentation of the Fetus, Multiple Pregnancy and Excess Amniotic Fluid, Cervical Incompetence, Trauma to the Abdomen [4] [5].

\section{C) Diagnosis of PROM}

When there is a rupture in the Fetal membranes, the woman notices a painless sudden leakage of fluid from her vagina, which is usually excess and watery. However, when the amount of amniotic fluid in the sac is minimal, the leaking fluid may only wet her underwear, and you may be unsure whether to make the diagnosis of PROM from the woman's complain [6].

\section{D) Complications of PROM}

PROM is associated with several potentially life-threatening complications: Cord Prolapse Fetal Hypoxia and Asphyxia, Placental Abruption [7]-[9].

\subsection{Aim of the Study}

To describe the characteristics of patients with PROM in Al Gumhoria Hospital.

To calculate the frequency of potential risk factors of PROM.

To calculate the frequency of methods used in management of PROM.

To calculate the frequency of fetal and maternal complications of PROM.

To put guidelines for early diagnosis and management of PROM.

To test statistical difference between proportions doing C/S by gestational age and maternal age. Chi-square was used and $\mathrm{P}$ value $<0.05$ was considered significant

\subsection{Material and Methods of the Study}

A total of 100 patients admitted to Al Gumhoria Hospital diagnosed with premature rapture of membranes. The Study was conducted in compliance with University of Benghazi Review Board In The Period from $1^{\text {st }}$ January 2012-31 $1^{\text {st }}$ Dec 2012

\subsubsection{Inclusion Criteria}

Confirm Gestational age by ultrasonographic examination before 24 weeks of pregnancy. Diagnosis of PROM confirmed by clinical features and sterile speculum examination. Absence of labour at admission. Singleton cephalic pregnancy.

\subsubsection{Exclusion Criteria}

Uterine contraction. Multiple pregnancy, Polyhydramios Malpresentation Incompetent cervix, All cases with 
previous digital examination

Cases with history of sexual intercourse in the last 24 hrs.

Patients who met the inclusion criteria constituted the subjects of the study.

A pre-designed questionnaire was filled and physically examined and sterile Cusco speculum conducted, which aided in the collection of endocervical and posterior vaginal fornix swabs from all of them. Each swab was included on to blood, chocolate, Mac Conkey, and around dextrose agar plates, while chocolate agar plates were incubated in candle extinction and all other plates were incubated in air at 37 c for 24 - $48 \mathrm{hrs}$.

Wet preparation slides were also made from all swabs and examined for fungal elements. And a Gram stain film of all specimens was also examined for intracellular Gram-negative diplococci. Isolates that appeared as Gram variable coccobacilli oxidase negative and bacitracin positive were identified as Gardenella vaginalis. Other significant isolates were identified by conventional methods

The in vitro sensitivity test of some of the isolates was determined in Nuller-Hinton agar plates by standard method against Ampicillin (10 mg), Erythromycin (10 mg), Cloxacillin (5 mg), Gentamycin (10 mg) and Azithromycin (10 mg). Anaerobic facilities are lacking.

\section{Results}

This study was done In the Period from $1^{\text {st }}$ January 2012-31 $1^{\text {st }}$ Dec 2012, included 100 cases of PROM. Their mean age is 28.6 years, std. devation is 5.9 years, median is 28 years and range is $17-42$.

Table 1 shows the age distribution of 100 cases of PROM. The majority were at the age group of 21 - 30 .

Table 2 shows the education distribution of 100 cases of PROM; where university education was the most frequent forming $39 \%$ followed by secondary level $31 \%$ and preparatory level $21 \%$.

Table 3 shows the occupation distribution of 100 cases of PROM; almost $75 \%$ were housewives.

Table 4 shows the distribution of patients according to gravidity: primigravida were $48 \%$ followed by multipara $46 \%$, nulipara was reported among abortions were only reported among $17 \%$.

Table 5 shows the distribution of 100 cases with PROM according to hospital stay where $63 \%$ stayed for 1 - 3 days and $31 \%$ for 4 days and only 6\% stayed for more than one week, $83 \%$ of them were at gestational age 36 41 week and $92 \%$ had early booking .There was statistical significant difference between $\mathrm{C} / \mathrm{S}$ and vaginal delivery in the gestational age; $39.3 \%$ who reported C/S were $<37$ weeks compared to $18.1 \%$ who reported vaginal delivery $\left(\chi^{2}=6.4\right.$. $\mathrm{P}$ value, 0.05$)$.

Table 6 shows the distribution of duration and suspected causes of leakage where $83 \%$ occurred more than 24

Table 1. Distribution of patients according to age.

\begin{tabular}{ccc}
\hline Age & No. & $\%$ \\
\hline 20 & 4 & 4 \\
$21-30$ & 61 & 61 \\
$31-40$ & 31 & 31 \\
$>40$ & 4 & 4 \\
Total & 100 & 100 \\
\hline
\end{tabular}

Table 2. Distribution of patients according to level of education.

\begin{tabular}{ccc}
\hline Level of Education & Number & \% \\
\hline Illiterate & 2 & 2 \\
Preparatory & 21 & 21 \\
Primary & 7 & 7 \\
Secondary & 31 & 31 \\
University & 39 & 39 \\
Total & 100 & 100 \\
\hline
\end{tabular}


Table 3. Distribution of patients according to occupation.

\begin{tabular}{ccc}
\hline Occupation & Number & \% \\
\hline Housewife & 75 & 75 \\
Employer & 22 & 22 \\
Student & 3 & 3 \\
Total & 100 & 100 \\
\hline
\end{tabular}

Table 4. Distribution of patients according to obstetrics history.

\begin{tabular}{ccc}
\hline Gravity & Number & \% \\
\hline Primi & 48 & 48 \\
$2-4$ & 46 & 46 \\
$\geq 5$ & 6 & 6 \\
Parity & Number & \% \\
Nullipara & 52 & 52 \\
$1-4$ & 44 & 44 \\
$\geq 5$ & 4 & 4 \\
Abortion & Number & \% \\
No abortion & 80 & 80 \\
One & 15 & 15 \\
Two & 5 & 5 \\
Total & 100 & 100 \\
\hline
\end{tabular}

Table 5. Distribution of patients according to according to hospital stay \& antenatal care history.

\begin{tabular}{ccc}
\hline Duration of hospital stay & Number & \% \\
\hline 1 - 3 days & 63 & 63 \\
4 - 6 days & 31 & 31 \\
$\geq 7$ days & 6 & 6 \\
Gestational age in weeks & Number & $\mathbf{\%}$ \\
30 - 36 & 17 & 17 \\
37 - 41 & 83 & 83 \\
Booking & Number & $\mathbf{\%}$ \\
Early & 92 & 92 \\
Late & 8 & 8 \\
Total & 100 & 100 \\
\hline
\end{tabular}


Table 6. Distribution of patients by duration and suspected causes of leaking.

\begin{tabular}{ccc}
\hline Duration of leaking hours & Number & $\%$ \\
\hline$\leq 24$ & 17 & 17 \\
$>24$ & 83 & 83 \\
Suspected causes of leaking & Number & $\%$ \\
Unknown & 43 & 43 \\
Previous PROM & 26 & 26 \\
Underling causes of Itching & 14 & 14 \\
Recurrent UTI & 12 & 12 \\
Polyhydramnios & 1 & 1 \\
Unstable lie & 1 & 1 \\
Cervical incompetence & 1 & 1 \\
Antepartum hemorrhage & 1 & 1 \\
Fever & 1 & 1 \\
Total & 100 & 100 \\
\hline
\end{tabular}

hours and high percentage (43\%) were due to unknown causes and $26 \%$ had previous PROM, $14 \%$ caused by itching and $12 \%$ by recurrent UTI.

Table 7 shows the distribution of 100 PROM cases according to temperature where only $4 \%$ of them were feverish, $55 \%$ had pulse more than 80 /minute and $10 \%$ of them had bp $>120 \mathrm{mHg}$.

Table 8 shows the distribution of patients according to type of treatment given to the mother where $66.1 \%$ have received antibiotics, $32.1 \%$ received dexamethazone and only $1.7 \%$ received tocolytics.

Table 9 shows the distribution of patients according to swabs results. The results were positive among $8 \%$ of endocervical swab and among $23 \%$ posterior vaginal fornix swabs.

Table 10 shows the distribution of patients according to mode of delivery full term normal delivery occurred in $62 \%$ of cases followed by $28 \%$ for C/S and only $10 \%$ were post term normal delivery. Almost, $50 \%$ of C/S indication was failed induction and fetal distress along with slow fetal heart accounted 14.3\%. Big size baby accounted for $3.6 \%$ similar to previous $2 \mathrm{C} / \mathrm{S}$, old scar, leaked liquor.

$\mathrm{C} / \mathrm{S}$ were significantly different in age group $<22$ years: all $\mathrm{C} / \mathrm{S}$ reported compared to $77.8 \%$ reported vaginal delivery $\left(\chi^{2}=5\right.$ o, $\left.\mathrm{P}, 0.02\right)$. Only $4.8 \%$ who reported FTND had gestational age $<37$ weeks compared to $39.3 \%$ who reported $\mathrm{C} / \mathrm{S}$ with significant difference $\left(\chi^{2}=47\right.$. P, 0.001).

Table 11 shows Distribution according the isolated microorganism where Candida albicans was the most frequent accounting for $40 \%$ followed by G. Vaginal (25\%) and antibiotic sensitivity was most frequently reported for Erythromycin accounting $21.7 \%, 17.4 \%$ for azithromycin and $15.9 \%$ for Ofloxacin

Table 12 shows the distribution of babies according to Apgar score and development of complications: Apgar score of $\%$ was the most frequent accounting for $79 \%$ followed by 4 accounting for $17 \%$ and only $1 \%$ complication had been reported.

\section{Discussion}

This descriptive study was done in Benghazi teaching hospital during 2012 on 100 subjects with singleton pregnancy. The results of the study match the findings of other local and overseas studies in the prevalence of risk factors such as younger age, high risk pregnancy as an indication for C/S rates of C/S, antibiotics, Dexamethasones.

\subsection{Characteristics of the Study Subjects}

The mean age of the patients was 28.6 years with singleton pregnancy with similar age in studies done by 
Table 7. Distribution of patients by measurements of physical signs.

\begin{tabular}{ccc}
\hline Measurement of Temperature & Number & \% \\
\hline $37^{\circ} \mathrm{C}$ & 96 & 96 \\
$>37^{\circ} \mathrm{C}$ & 4 & 4 \\
Measurement of Pulse/minute & Number & \% \\
$\leq 80$ & 45 & 45 \\
$>80$ & 55 & 55 \\
Systolic Blood Pressure & Number & \% \\
$\leq 120 \mathrm{mmHg}$ & 90 & 90 \\
$>120 \mathrm{mmHg}$ & 10 & 10 \\
Total & 100 & 100 \\
\hline
\end{tabular}

Table 8. Distribution of patients by type of treatment to the mother.

\begin{tabular}{ccc}
\hline Type of Treatment & Number & \% \\
\hline Antibiotic & 39 & 66.1 \\
Dexamethasone & 19 & 32.2 \\
Tocolytic & 1 & 1.7 \\
Total & 59 & 100 \\
\hline
\end{tabular}

Table 9. Distribution of patients by gynaecological examination.

\begin{tabular}{ccc}
\hline Fundal Level & Number & \% \\
\hline Corresponds to Date & 77 & 77 \\
Decreased & 23 & 23 \\
Vulval Examination & Number & \% \\
Normal & 92 & 92 \\
Abnormal & 8 & 8 \\
Vaginal Examination & Number & \% \\
Normal & 90 & 90 \\
Abnormal & 10 & 10 \\
Cervical Examination & Number & \% \\
Normal & 99 & 99 \\
Abnormal & 1 & 1 \\
Total & 100 & 100 \\
\hline
\end{tabular}

Alhussini in Egypt and Saudi Arabia, the cases of the Pakistani study done by Noor S. were younger” 15 - 25 years" [10] [11]. Premature PROM was reported among $10 \%$ of the cases in this study which is more or less comparable to PPROM reported by Noor was $9.5 \%$ because of younger age as well as low socioeconomic class compared to findings of SmithG and Tahir i.e. 2.3\% and 5.4\% respectively [10] [12].

The gestational age more than 36 weeks was reported in $83 \%$ of the study subjects with $>92 \%$ with early booking and only $6 \%$ were hospitalized for more than a week. Almost $83 \%$ of the patients reported leakage 
Table 10. Distribution of patients by mode of delivery and indication of Caesarean section.

\begin{tabular}{ccc}
\hline Mode of Delivery & Number & $\%$ \\
\hline FTND & 62 & 62 \\
PTNVD & 10 & 10 \\
C/S & 28 & 28 \\
Indication of C/S & Number & $\%$ \\
Failed Induction & 14 & 50 \\
Fetal Distress & 4 & 14.3 \\
Slow Fetal Heart & 4 & 14.3 \\
Big Size Baby & 1 & 3.6 \\
Previous Scar & 1 & 3.6 \\
Drained Liquor & 1 & 3.6 \\
Previous 2 C/S & 1 & 3.6 \\
Breech Presentation & 2 & 7.2 \\
Total & 100 & 100 \\
\hline
\end{tabular}

Table 11. Distribution by isolated microorganism and antibiotic sensitivity.

\begin{tabular}{ccc}
\hline Microorganism Isolated from Leaking Water & Number & $\%$ \\
\hline Candida albicans & 8 & 40 \\
Gardenella vaginalis & 5 & 25 \\
Staphylococcus aureus & 2 & 10 \\
Streptococcus pyogenes & 2 & 10 \\
CONS & 2 & 10 \\
Streptococcus pneumonia & 1 & 5 \\
Total & 20 & 100 \\
Type of Antibiotic & Number & $\%$ \\
Erythromycin & 15 & 21.7 \\
Azithromycin & 12 & 17.4 \\
Ofloxacin & 11 & 15.9 \\
Gentamycin & 9 & 13 \\
Ampicillin & 6 & 8.7 \\
Cloxacil & 69 & 8.7 \\
Cefuroxime & 5.3 \\
Chloramphenicol & 5 & 100 \\
Total & 5.3 & \\
\hline & 5 & 7.3 \\
\hline
\end{tabular}

more than 24 hours. Primi gravidity was reported among 48\% and Nuliparity was reported among 52\% of the study subjects which are comparable to studies done in the region by al husseni, Noor and Tahir [10] [11] [13] demonstrating high frequency of PROM among nuliparous women compared to parous ones The most frequent suspected causes of PROM in the study were previous PROM, itching, recurrent UTI accounting for 26\%, $14 \%$ 
Table 12. Distribution of babies by Apgar score and complications.

\begin{tabular}{ccc}
\hline Apgar Score & Number & \% \\
\hline 0 & 1 & 1 \\
4 & 17 & 17 \\
5 & 79 & 79 \\
Not Recorded & 3 & 3 \\
Complication & Number & $\%$ \\
Yes & 1 & 1 \\
No & 99 & 99 \\
Total & 100 & 100 \\
\hline
\end{tabular}

and $12 \%$ respectively $43 \%$ were of unknown causes. polyhydrominous. Unstable lie, cervical incompetence, Antipartum haemorrhage and fever were the least reported causes and each was $1 \%$. Maternal infection was the most frequent cause of PROM in the study done by Modena in Italy 2004 [14] as well as smoking of the pregnant women and Cocaine intake which is the case in our study at all. But, Asindi reported high frequency of infections with CONS in Saudi Arabia and 24\% of the mothers were colonized and 31\% of their babies [15]. Mercer in his recent expert view 2003 concluded that PROM is a multifactorial in nature, and choridecidual infection or inflammation appear to play important role in the aetiology of preterm PROM specially in early gestational age. He also reported decreased membrane collagen content that increased with the gestational age and was associated with the pathogenesis of the condition [16]. Our study agree with the findings of Mercer in the suspected causes of PROM like prior conditions, uterine distension, infections, cervical incompetence and ante partum haemorrhage but he reported cigarette smoking, amniocentesis, and prior cervical conisation.

\subsection{Management and Mode of Delivery}

The swabs were done for the studies subjects showed that only $8 \%$ of the endocervical swabs were positive and $23 \%$ of the posterior vaginal fornix with similar findings from the study done by AlHussani in Assuit, Egypt 2012 who reported positive culture among cases of PROM significantly higher than among their matched control and the presence of significant association with the educational level, residence and education [10]. The most frequent micro organism in the current study was Candida albicans forming $40 \%$ followed by Gardenella vaginalis for $25 \%$ and CONS for $10 \%$ like Staphylococcus aureus and streptococcus pyogenous Although, the current study findings are in consistence with the done by Alhussani in Egypt they are not comparable to the findings of the study done by Asindi in Saudi Arabia who reported the frequency of CONS as high as 31\% and Candida was the least frequent orgnasim [10] [15]. On the contrary, Karat et al. 2006 in case control study reported rates of micro organisms in cases of PROM and their matched controls as: $16 \%$ - 28\% for Staph aureus, 8\% - 19\% for Candida albicans, and 3.3\% - 20\% for E. coli these non matched results could be explained by the variability of sociodemographic characteristics, educational levels and racial origins [17].

Dexamethasone was administered to $32.2 \%$ of the subjects aiming to decreasing perinatal morbidity and mortality, this procedure is consistence with the guidlines subjected by Harding JE as a result of his meta analysis that concluded reduced risk of RDS and IVH and necrotising entercolitis among those receiving corticosteroids versus no administration as all physicians caring for pregnant women should understand the dosing and indications for corticosteroid administration during pregnancy [18]. The most widely used and recommended regimens include intramuscular dexamethasone (Decadron) $6 \mathrm{mg}$ every 12 hours for two days [19]. Other centers use intramuscular betamethasone (Celestone) $12 \mathrm{mg}$ every 24 hours for two days, The National Institutes of Health recommends administration of corticosteroids before 30 to 32 weeks' gestation, assuming fetal viability and no evidence of intra-amniotic infection. Use of corticosteroids between 32 and 34 weeks is controversial [14]. Tocolysis was only conducted in $2.7 \%$ of the cases. Limited data are available to help to determine whether tocolyis is effective in the management of PROM, Tocolytic therapy can prolong latent period by inhibition of uterine contraction but did not proved to improve neonaltal survival

The mode of delivery of the cases was as follow $62 \%$ by FTNVD and $10 \%$ by PTNV, Caesarean section was 
indicated for $28 \%$ of cases $50 \%$ of the indications were due to failure of induction, $14.3 \%$ were reported equally to fetal stress and slow fetal heart rate, $3.6 \%$ of cases was reported for six indications; macrosomia, old scar, breech presentation, previous $2 \mathrm{~S} / \mathrm{C}$, drained liquor a. The rates in this study were similar to what was reported by Elsalmani and his colleagues in Iran 2002, where 28.06\% were subjected to C/S with higher rate among nullipara compared to Multipara [20]. Noor in Pakistan reported 14\% rate of C/S [11]. The highest reports rates for $\mathrm{C} / \mathrm{S}$ were reported in developing countries.

\subsection{Foetomaternal Outcomes}

A single born baby had complication by PROM forming $1 \%$ compared to higher figures reported by Noor as high neonatal mortality assumed to be due younger gestational age $<33-36$ or due to low socioeconomic status with higher rates of infections and low birth weights [11].

\section{Recommendations}

\subsection{Non-Specific Recommendations}

Larger analytical studies should be done.

\subsection{Specific Recommendations}

Erythromycin (250 mg orally 6 hourly) should be given for 10 days following the diagnosis of PPROM from 20 weeks' gestation only if theres no clinical evidence of only if there no clinical evidence of chorioamnionitis or maternal sepsis. Women with PPROM are at increased risk of infection. Oral erythromycin is indicated as antibiotic prophylaxis only. If there is clinical evidence of chorioamnionitis or maternal sepsis, a septic work-up should be obtained and broad spectrum intravenous antibiotics commenced. The choice of antibiotics used can be determined locally but should include appropriate cover for GBS, E. coli, Listeria and anaerobes. Delivery is indicated in the management of chorioamnionitis. Co-amoxiclav is not recommended for women with PPROM because of concerns about necrotizing enterocolitis. There was also a significant reduction in the number of babies with an abnormal cerebral ultrasound scan prior to discharge from. This review shows that routine antibiotic administration to women with PPROM reduces some markers of maternal and neonatal morbidity. This does not translate into a significant reduction in perinatal mortality. The authors conclude that the decision to prescribe antibiotics is not clear-cut. Although antibiotic administration following PPROM is associated with neonatal benefits this is not translated to either benefit or harm with longer term seven year follow-up. Benefits in short term outcomes should be balanced against a lack of evidence of benefit for perinatal mortality and long term outcome. If antibiotics are prescribed, it is unclear which would be the antibiotic of choice. Co-amoxiclav should be avoided because of the increased risk of neonatal necrotizing enterocolitis. The Cochrane collaboration previously presented results on antibiotic use in 2005. There was a variation in the choice of antibiotics used and the duration of therapy in the studies examined in the meta-analysis Ten trials tested broad spectrum penicillin, either alone or in combination, five tested macrolide antibiotics (erythromycin) either alone or in combination and one trial tested clindamycin and gentamycin. The duration of treatment varied between two doses and 10 days. Any penicillin (except co-amoxiclav) or erythromycin versus placebo was associated with a significant reduction in the numbers of babies born within 48 hours and who had positive blood cultures. Co-amoxiclav versus placebo was associated with an increase in the numbers of babies born with necrotizing enterocolitis. On balance, it would seem reasonable to prescribe prophylactic erythromycin because of the reduction in some markers of maternal and neonatal morbidity. The view of this guideline is that it is reasonable to prescribe antibiotics from 20 weeks gestation. In addition the risk of intrauterine infection is higher in early gestations partly because of the less well developed antimicrobial properties of amniotic fluid. Therefore oral erythromycin is indicated as antibiotic prophylaxis. Women with PPROM are at increased risk of infection. Oral erythromycin is indicated as antibiotic prophylaxis only. If there is clinical evidence of chorioamnionitis or maternal sepsis, a septic work-up should be obtained and broad spectrum intravenous antibiotics commenced. The choice of antibiotics used can be determined locally but should include appropriate cover for GBS, E. coli, Listeria and anaerobes. Delivery is also indicated in the management of chorioamnionitis. If Group B streptococcus is isolated in cases of PPROM, antibiotics should be given in line with the recommendations for routine intra-partum prophylaxis. 


\subsection{The Role of Antenatal Corticosteroids}

Antenatal corticosteroid should be administered in women with PROM. Indications for antenatal corticosteroid therapy include women with PPROM between 24 and 34 weeks' gestation. Prophylactic tocolysis in women with PPROM without uterine activity is not recommended.

Women with PPROM and uterine activity who require intrauterine transfer or antenatal corticosteroids may be considered for a short course of tocolysis. However, this decision needs to be considered in the light of the possibility of pre-existing intrauterine infection, the only clinical feature of which might be uterine activity. If there is a significant suspicion of chorioamnionitis, then tocolysis is not recommended.

\subsection{Prophylactic Tocolysis}

Three randomised studies on a total of 235 patients with PPROM reported that the proportion of women remaining undelivered 10 days after membrane rupture was not significantly higher in those receiving tocolysis compared to those receiving none (How, 1998; Levy and Warsof, 1985; Dunlop, 1986). A retrospective casecontrol study showed that tocolysis after PPROM did not increase the interval between membrane rupture and delivery or reduce neonatal morbidity (Jazayeri, 2003).

\subsection{Therapeutic Tocolysis}

Tocolysis aims not only to inhibit uterine contractions but also to allow a safe transfer of the pregnant patient to a tertiary care centre. It gives the opportunity to administrate corticosteroids for preventing neonatal risks associated with prematurity.

\section{References}

[1] Mercer, B.M., Goldenberg, R.L., Meis, P.J., et al. (2000) The Preterm Prediction Study: Prediction of Preterm Premature Rupture of the Membranes Using Clinical Findings and Ancillary Testing. American Journal of Obstetrics \& Gynecology, 183, 738-745.

[2] Mercer, B.M., Moretti, M.L., Prevost, R.R., et al. (1992) Erythromycin Therapy in Preterm Premature Rupture of the Membranes: A Prospective, Randomized Trial of 220 Patients. American Journal of Obstetrics \& Gynecology, 166, 794-802. http://dx.doi.org/10.1016/0002-9378(92)91336-9

[3] Johnson, J.W.C., Egerman, R.S. and Moorhead, J. (1990) Cases with Ruptured Membranes That "Reseal". American Journal of Obstetrics \& Gynecology, 163, 1024-1030. http://dx.doi.org/10.1016/0002-9378(90)91117-U

[4] Silver, R.K., MacGregor, S.N. and Hobart, E.D. (1989) Impact of Residual Amniotic Fluid Volume in Patients Receiving Parenteral Tocolysis after Premature Rupture of the Membranes. American Journal of Obstetrics \& Gynecology, 161, 784-787. http://dx.doi.org/10.1016/0002-9378(89)90402-X

[5] VIntzileos, A.M., Campbell, W.A., Nochimson, D.J., et al. (1986) Qualitative Amniotic Fluid Volume versus Amniocentesis in Predicting Infection in Preterm Premature Rupture of the Membranes. Obstetrics \& Gynecology, 67, 579583.

[6] Mercer, B.M. (2003) Premature Rupture of the Membranes. An Expert’s View. Obstetrics \& Gynecology, 101, 178193. http://dx.doi.org/10.1016/S0029-7844(02)02366-9

[7] Lonky, N.M. and Hayashi, R.H. (1988) A Proposed Mechanism for Pre Mature Rupture of the Membranes. Obstetrical \& Gynecological Survey, 43, 22-28. http://dx.doi.org/10.1097/00006254-198801000-00003

[8] Omero, R., Yoon, B.H., Mazor, M., et al. (1993) A Comparative Study of the Diagnostic Performance of Amniotic Fluid Glucose, White Blood Cell Count, IL 6, and Gram Stain in the Detection of Microbial Invasion in Patients with Preterm Premature Rupture of Membranes. American Journal of Obstetrics \& Gynecology, 169, 839-851. http://dx.doi.org/10.1016/0002-9378(93)90014-A

[9] Vintzileos, A.M., Bors Koefoed, R., Pelegano, I.F., et al. (1987) The Use of Fetal Biophysical Profile Improves Pregnancy Outcome in Premature Rupture of the Membranes. American Journal of Obstetrics \& Gynecology, 157, $236-240$. http://dx.doi.org/10.1016/S0002-9378(87)80141-2

[10] Al-Hussini, T.K., Mohamed, S.N., Alam Eldin, H. and Ahmed, A. (2012) Cervicovaginal Infection during Pregnancy and Its Relation to Preterm pre Labour Rupture of Membranes. The Journal of American Science, 18, 364-373.

[11] Noor, S., Fawwad, A., Shahzd, H., Sultanna, R. and Bashir, R. (2010) Foetomaternal Outcome in Patients with or without Premature Rupture of Membranes. Journal of Ayub Medical College, Abbottabad: JAMC, 22, 164-167.

[12] Smith, G., Rafuse, C., Anand, N., Brennan, B., Connors, G., Crane, J., et al. (2005) Prevalence, Management and Out- 
comes of Preterm Prelabour Rupture of the Membranes of Women in Canada. Journal of Obstetrics and Gynaecology Canada, 27, 547-553.

[13] Tahir, S., Aleem, A. and Aziz, R. (2001) Incidence and Outcome of Premature Preterm Rupture of Membranes. Pakistan Journal of Medical Sciences, 18, 26-32.

[14] Modena, A.B., Kaihura, C. and Fieni, S. (2004) Prelabour Rupture of the Membranes: Recent Evidence. Acta Biomedica, 75, 5-10.

[15] Asindi, A., Archibong, E.I. and Mannan, A.B. (1999) Neonatal Septicaemia. Saudi Medical Journal, 20, 924-948.

[16] Mercer, B.M. (2003) Preterm Premature Rupture of the Membranes: An Expert’s View. Obstetrics \& Gynecology, 101, 178-193. http://dx.doi.org/10.1016/S0029-7844(02)02366-9

[17] Karat, C., Madhivanan, P., Krupp, K., Pornima, S., Jayanthi, N.V., Suguna, J.S. and Mathai, E. (2002) The Clinical and Microbiological Correlates of Premature Rupture of Membranes. Indian Journal of Medical Microbiology, 24, $283-285$. http://dx.doi.org/10.4103/0255-0857.29388

[18] Harding, J.E., Pang, J., Knight, D.B. and Liggins, G.C. (2001) Do Antenatal Corticosteroids Help in the Setting of Preterm Ruprure of Membranes? American Journal of Obstetrics \& Gynecology, 184, 131-139.

[19] Mitchell, T.F., Pearlman, M.D., Chapman, R.L., et al. (2001) Maternal and Transplacental Pharmacokinetics of Cefazolin. Obstetrics \& Gynecology, 98, 1075-1079. http://dx.doi.org/10.1016/s0029-7844(01)01629-5

[20] Elsalmani, L. and Asadi, M. (2002) The Caesarean Section Rate in Cases with Premature Rupture of Membranes (PROM) at 36th Week of Pregnancy. Acta Medica Iranica, 40, 83-87.

\section{Abbreviation}

PROM: $\quad$ Premature Rupture of Membranes,

PPROM: $\quad$ Preterm Premature Rupture of Membrane,

CONS: Coagulase Negative Staphylococcus,

UUS: Uterine Ultrasound,

C/S: $\quad$ Caesarean Section,

FTND: $\quad$ Full term Normal Delivery,

PTNVD: $\quad$ Pre Term Full term Normal Delivery,

$\beta$ AdRA: $\quad \beta$ Adrenergic Receptor Agonist,

$\mathrm{MgSO}_{4}$ : $\quad$ Magnesium Sulphate,

PgSI: Prostaglandin Synthesis Inhibitor,

$\mathrm{Ca}^{++} \mathrm{CB}$ : Calcium Channel Blocker,

GBS: Group B Streptococcus (Streptococcus agalactiae). 